\title{
Operational experience of grid-integrated vehicles with V2G in three countries
}

Prof. Dr. Willett Kempton, Center for Carbon-free Power Integration, University of Delaware,

S. Fisher, NRG EVgo;

S. Fisher, PJM Interconnection;

G. Poilasne, Nuvve Corporation, USA

This manuscript was not available on completion of this publication. Thank you for your understanding. 